\title{
Acid Corrosion Inhibition and Adsorption Behaviour of Ethyl Hydroxyethyl Cellulose on Mild Steel Corrosion
}

\author{
I. O. Arukalam, ${ }^{1}$ I. O. Madu, ${ }^{1}$ N. T. Ijomah, ${ }^{1}$ C. M. Ewulonu, ${ }^{2}$ and G. N. Onyeagoro ${ }^{1}$ \\ ${ }^{1}$ Department of Polymer and Textile Engineering, Federal University of Technology, P.M.B. 1526, Owerri, Nigeria \\ ${ }^{2}$ Department of Polymer and Textile Engineering, Nnamdi Azikiwe University, P.M.B. 5025, Awka, Nigeria \\ Correspondence should be addressed to I. O. Arukalam; innocentarukalam@yahoo.com
}

Received 21 December 2013; Accepted 5 February 2014; Published 13 March 2014

Academic Editor: Jean-Francois Gohy

Copyright (c) 2014 I. O. Arukalam et al. This is an open access article distributed under the Creative Commons Attribution License, which permits unrestricted use, distribution, and reproduction in any medium, provided the original work is properly cited.

\begin{abstract}
The corrosion inhibition of mild steel in $1.0 \mathrm{M} \mathrm{H}_{2} \mathrm{SO}_{4}$ solution by ethyl hydroxyethyl cellulose has been studied in relation to the concentration of the additive using weight loss measurement, EIS, polarization, and quantum chemical calculation techniques. The results indicate that EHEC inhibited corrosion reaction in the acid medium and inhibition efficiency increased with EHEC concentration. Further increase in inhibition efficiency is observed in the presence of iodide ions, due to synergistic effect. Impedance results reveal that EHEC is adsorbed on the corroding metal surface. Adsorption followed a modified Langmuir isotherm, with very high negative values of the free energy of adsorption $\left(\Delta G_{\text {ads }}\right)$. The polarization data indicate that the inhibitor was of mixed type, with predominant effect on the cathodic partial reaction. The frontier molecular orbitals, HOMO (the highest occupied molecular orbital) and LUMO (the lowest unoccupied molecular orbital) as well as local reactivity of the EHEC molecule, were analyzed theoretically using the density functional theory to explain the adsorption characteristics at a molecular level. The theoretical predictions showed good agreement with experimental results.
\end{abstract}

\section{Introduction}

Organic compounds containing polar functional groups such as nitrogen, sulphur, and/or oxygen in a conjugated system have been reported to be effective as corrosion inhibitors for steel [1-8]. Some of the organic compounds are polymeric in nature and therefore possess multiple active centres. The study of corrosion inhibition by polymers has been on the increase in recent times. Polymers are employed as corrosion inhibitors because the presence of many adsorption centres helps them form complexes with metal ions. The formed complexes were adsorbed on the metal surface to form a barrier film which separated the metal surface from the corrosive agents present in the aggressive solution [9-14]. The effectiveness of inhibition by the adsorbed inhibitor system will be determined by the energy released on forming the metal-inhibitor bond compared to the corresponding changes when the pure acid reacts with the metal [15].

Some authors have reported on the effectiveness of polymeric corrosion inhibitors [16-20]. In their accounts, the inhibitive power of these polymers is related structurally to the cyclic rings and heteroatoms which are the major active centres of adsorption.

In order to support experimental studies, theoretical calculations are conducted in order to provide molecularlevel understanding of the observed experimental behaviour. The major driving force of quantum chemical research is to understand and explain the functions of ethyl hydroxyethyl cellulose in molecular forms. Among quantum chemical methods for evaluation of corrosion inhibitors, density functional theory (DFT) has shown significant promise [21-23] and appears to be adequate for pointing out the changes in electronic structure responsible for inhibitory action. The geometry of the inhibitor is in ground state, as well as the nature of their molecular orbitals, HOMO (the highest occupied molecular orbital) and LUMO (the lowest unoccupied molecular orbital), that are involved in the properties of activity of inhibitors $[24,25]$.

The present study presents the appraisal of inhibitive capability of ethyl hydroxyethyl cellulose (EHEC) on mild 
steel corrosion in $1.0 \mathrm{M} \mathrm{H}_{2} \mathrm{SO}_{4}$ solution using weight loss measurements and quantum chemical calculations techniques.

\section{Materials and Methods}

2.1. Sample Preparation. Tests were performed on mild steel specimens of the following percentage chemical composition: Si: 0.02; C: 0.05; Mn: 0.18; Cu: 0.02; Cr: 0.02 and the remainder $\mathrm{Fe}$. This was machined into test coupons of dimensions $3 \times$ $2 \times 0.05 \mathrm{~cm}$ and a small hole drilled at one end of the coupon to enable suspension into the test solution in the beaker. The metal specimens were polished with fine emery paper, degreased, and cleaned as described elsewhere [26, 27]. EHEC sourced from Sigma Aldrich chemical company was used without further purification at concentrations of $0.5,1.0,1.5,2.0$, and $2.5 \mathrm{~g} / \mathrm{L}$. Blank sulphuric acid solution was prepared in the concentration of $1.0 \mathrm{M} \mathrm{H}_{2} \mathrm{SO}_{4}$. The potassium iodide, KI from BDH Laboratory Supplies, was used. $0.5 \mathrm{~g} / \mathrm{L} \mathrm{KI}$ was prepared and added to each of the solutions containing the additive.

2.2. Weight Loss Measurements. Weight loss experiments were conducted on test coupons. Tests were conducted under total immersion conditions in $200 \mathrm{~mL}$ of test solutions at ambient temperature, $28 \pm 1^{\circ} \mathrm{C}$. The pre-cleaned and weighed coupons were suspended in beakers containing the solutions using glass rods and hooks. All tests were made in aerated solutions and were run three times to ensure reproducibility. To determine weight loss with respect to time, the coupons were retrieved from test solutions at $24 \mathrm{~h}$ intervals progressively for $120 \mathrm{~h}$ ( 5 days). At the end of the tests, the weight loss was taken to be the mean value of the difference between the initial and final weights of the coupons for the three determinations at a given time. The corrosion rates of mild steel in $1.0 \mathrm{M} \mathrm{H}_{2} \mathrm{SO}_{4}$ solution and the acid solution containing the additive, EHEC, were calculated from the expression:

$$
\text { Corrosion rate, } R_{c}(\mathrm{~mm} / \mathrm{y})=\left[\frac{87,600 \Delta W}{\rho A t}\right] \text {, }
$$

where $\Delta W, \rho, A, t$ are weight loss in gram, density of mild steel in $\mathrm{g} / \mathrm{cm}^{3}$, surface of the test coupon in $\mathrm{cm}^{2}$, and time period of exposure in the test solution in hour, respectively.

2.3. Electrochemical Experiments. Electrochemical experiments were performed using a VERSASTAT 3 Advanced Electrochemical System operated with V3 Studio electrochemical software. A conventional three-electrode glass cell was used for the experiments. Test coupons with $1 \mathrm{~cm}^{2}$ exposed surface area were used as working electrode and a graphite rod as counterelectrode. The reference electrode was a saturated calomel electrode (SCE), which was connected via Luggin's capillary. The working electrode was immersed in a test solution for 30 minutes to attain a stable open circuit potential prior to electrochemical measurements. All experiments were undertaken in $300 \mathrm{~mL}$ of stagnant aerated solutions at $29 \pm 1^{\circ} \mathrm{C}$. Each test was run in triplicate to verify the reproducibility of the systems. Electrochemical impedance spectroscopy (EIS) measurements were made at corrosion potentials $\left(E_{\text {corr }}\right)$ over a frequency range of $100 \mathrm{kHz}-10 \mathrm{mHz}$, with a signal amplitude perturbation of $5 \mathrm{mV}$. Spectra analyses were performed using Zsimpwin software. Potentiodynamic polarization studies were carried out in the potential range -250 to $+250 \mathrm{mV}$ at a scan rate of $0.33 \mathrm{mV} \mathrm{s}^{-1}$.

All theoretical quantum chemical calculations were performed using the density functional theory (DFT) electronic structure programs, Forcite and DMol3 as contained in the Materials Studio 4.0 software.

\section{Results and Discussion}

3.1. Corrosion Rates. The corrosion rates of metals and alloys in aggressive solutions can be determined using different electrochemical and nonelectrochemical techniques. The mechanism of anodic dissolution of iron in acidic solutions corresponds to [28]

$$
\begin{gathered}
\mathrm{Fe}+\mathrm{OH} \Longleftrightarrow \mathrm{FeOH}_{\mathrm{ads}}+e^{-} \\
\mathrm{FeOH}_{\mathrm{ads}} \longrightarrow \mathrm{FeOH}^{+}+e^{-} \\
\mathrm{FeOH}^{+}+\mathrm{H}^{+} \Longleftrightarrow \mathrm{F}^{2+}+\mathrm{H}_{2} \mathrm{O} .
\end{gathered}
$$

As a consequence of these reactions, including the high solubility of the corrosion products, the metal loses weight in the solution. The results of the gravimetric determination of mild steel corrosion rate as a function of time and concentration of the additive are given in Table 1.

These results show that the corrosion rate of mild steel in $1.0 \mathrm{M} \mathrm{H}_{2} \mathrm{SO}_{4}$ decreases with time in systems with additive and the blank acid solution. The effects of addition of different concentrations of EHEC on corrosion rates in the acid solution after 5 days of exposure are shown in Table 1. EHEC is observed to reduce the corrosion rate at the studied concentration of $0.5 \mathrm{~g} / \mathrm{L}$ EHEC, indicating inhibition of the corrosion reaction. This effect becomes more pronounced with increasing concentration of the inhibitor, which suggests that the inhibition process is sensitive to the concentration (amount) of the additive present.

3.2. Inhibition Efficiency. A quantitative evaluation of the effect of EHEC on mild steel corrosion in $1.0 \mathrm{M} \mathrm{H}_{2} \mathrm{SO}_{4}$ solution was achieved from appraisal of the inhibition efficiency (I\%) given by

$$
I \%=\left[1-\frac{R_{\mathrm{cinh}}}{R_{\mathrm{cblk}}}\right] \times 100,
$$

where $R_{\text {cinh }}$ and $R_{\text {cblk }}$ are the corrosion rates in inhibited and uninhibited solutions, respectively. The values obtained for the inhibition efficiency are given in Table 2.

The plots show that $I \%$ increased progressively with concentration of the additive (Figure 1). Following the observed trend of inhibition, organic inhibitors are known to decrease metal dissolution by forming a protective adsorption film 
TABLE 1: Calculated values of corrosion rate of mild steel in $1.0 \mathrm{M} \mathrm{H}_{2} \mathrm{SO}_{4}$ in the absence and presence of EHEC and KI.

\begin{tabular}{|c|c|c|c|c|c|}
\hline \multirow{3}{*}{ System } & \multicolumn{5}{|c|}{ Corrosion rate $(\mathrm{mm} / \mathrm{y})$} \\
\hline & \multicolumn{5}{|c|}{ Day } \\
\hline & 1 & 2 & 3 & 4 & 5 \\
\hline Blank & 25.27 & 22.36 & 20.16 & 19.03 & 17.99 \\
\hline $0.5 \mathrm{~g} / \mathrm{L}$ EHEC & 14.50 & 12.07 & 10.93 & 10.39 & 10.10 \\
\hline $0.5 \mathrm{~g} / \mathrm{L} \mathrm{EHEC}+\mathrm{KI}$ & 12.20 & 10.59 & 9.50 & 9.27 & 9.06 \\
\hline $1.0 \mathrm{~g} / \mathrm{L} \mathrm{EHEC}$ & 12.92 & 10.78 & 9.74 & 9.23 & 8.97 \\
\hline $1.0 \mathrm{~g} / \mathrm{L} \mathrm{EHEC} \mathrm{+} \mathrm{KI}$ & 9.39 & 7.97 & 7.25 & 7.17 & 7.19 \\
\hline $1.5 \mathrm{~g} / \mathrm{L}$ EHEC & 13.22 & 11.43 & 10.31 & 9.79 & 9.51 \\
\hline $1.5 \mathrm{~g} / \mathrm{L}$ EHEC + KI & 9.85 & 8.22 & 7.37 & 7.16 & 7.05 \\
\hline $2.0 \mathrm{~g} / \mathrm{L}$ EHEC & 11.84 & 10.08 & 9.20 & 8.79 & 8.59 \\
\hline $2.0 \mathrm{~g} / \mathrm{L} \mathrm{EHEC}+\mathrm{KI}$ & 10.47 & 8.37 & 7.31 & 6.81 & 6.60 \\
\hline $2.5 \mathrm{~g} / \mathrm{L}$ EHEC & 11.40 & 9.92 & 9.35 & 8.80 & 8.59 \\
\hline $2.5 \mathrm{~g} / \mathrm{L}$ EHEC + KI & 10.06 & 8.21 & 7.70 & 7.56 & 7.48 \\
\hline
\end{tabular}

TABLE 2: Calculated values of inhibition efficiency of mild steel in $1.0 \mathrm{M} \mathrm{H}_{2} \mathrm{SO}_{4}$ in the presence of EHEC and KI.

\begin{tabular}{|c|c|c|c|c|c|}
\hline \multirow{3}{*}{ System } & \multicolumn{5}{|c|}{ Inhibition efficiency $(I \%)$} \\
\hline & \multicolumn{5}{|c|}{ Day } \\
\hline & 1 & 2 & 3 & 4 & 5 \\
\hline $0.5 \mathrm{~g} / \mathrm{L}$ EHEC & 42.62 & 46.02 & 45.78 & 45.40 & 43.86 \\
\hline $0.5 \mathrm{~g} / \mathrm{L}$ EHEC + KI & 51.72 & 52.64 & 52.88 & 51.29 & 49.64 \\
\hline $1.0 \mathrm{~g} / \mathrm{L} \mathrm{EHEC}$ & 48.87 & 51.79 & 51.69 & 51.50 & 50.14 \\
\hline $1.0 \mathrm{~g} / \mathrm{L} \mathrm{EHEC} \mathrm{+} \mathrm{KI}$ & 62.84 & 64.36 & 64.04 & 62.32 & 60.03 \\
\hline $1.5 \mathrm{~g} / \mathrm{L} \mathrm{EHEC}$ & 47.69 & 48.88 & 48.86 & 48.55 & 47.14 \\
\hline $1.5 \mathrm{~g} / \mathrm{L}$ EHEC + KI & 61.02 & 63.24 & 63.44 & 62.38 & 60.81 \\
\hline $2.0 \mathrm{~g} / \mathrm{L}$ EHEC & 53.15 & 54.92 & 54.37 & 53.81 & 52.25 \\
\hline $2.0 \mathrm{~g} / \mathrm{L}$ EHEC + KI & 58.57 & 62.57 & 63.74 & 64.21 & 63.31 \\
\hline $2.5 \mathrm{~g} / \mathrm{L}$ EHEC & 54.89 & 55.64 & 53.62 & 53.76 & 52.25 \\
\hline $2.5 \mathrm{~g} / \mathrm{L} \mathrm{EHEC} \mathrm{+} \mathrm{KI}$ & 60.19 & 63.28 & 61.81 & 60.27 & 58.42 \\
\hline
\end{tabular}

which blocks the metal surface, separating it from the corrosive medium [29-32]. Consequently, in inhibited solutions, the corrosion rate is indicative of the number of free corroding sites remaining after some sites have been effectively blocked by inhibitor adsorption. It has been suggested [33, 34], however, that anions such as $\mathrm{Cl}^{-}, \mathrm{I}^{-}, \mathrm{SO}_{4}{ }^{2-}$, and $\mathrm{S}^{2-}$ may also participate in forming reaction intermediates on the corroding metal surface, which either inhibit or stimulate corrosion. It is important to recognize that the suppression or stimulation of the dissolution process is initiated by the specific adsorption of anion on the metal surface.

3.3. Effect of Halide Ion Addition. To further clarify the modes of inhibitor adsorption, experiments were conducted in the presence of iodide ions, which are strongly adsorbed on the surface of mild steel in acidic solution and facilitate adsorption of organic cation-type inhibitors by acting as intermediate bridges between the positive end of the organic cation and the positively charged metal surface. Specific adsorption of iodide ions on the metal surface leads to recharging the electrical double layer [35]. The inhibitor is then drawn into the double layer by electrostatic interaction with the adsorbed $\mathrm{I}^{-}$ions, forming ion pairs on the metal surface which increases the degree of surface coverage:

$$
\begin{aligned}
\mathrm{I}^{-}{ }_{\text {sol }} & \longrightarrow \mathrm{I}^{-} \text {ads } \\
\mathrm{I}^{-}{ }_{\text {ads }}+\mathrm{Inh}^{+}{ }_{\text {sol }} & \longrightarrow\left[\mathrm{I}^{-}-\mathrm{Inh}^{+}\right]_{\mathrm{ads}} .
\end{aligned}
$$

Thus, an improvement of $I \%$ on addition of KI is an indication of the participation of protonated inhibitor species in the adsorption process (Figure 2). Table 2 illustrates the effect of addition of $0.5 \mathrm{~g} / \mathrm{L} \mathrm{KI}$ to the different concentrations of EHEC on the corrosion of mild steel in $1.0 \mathrm{M} \mathrm{H}_{2} \mathrm{SO}_{4}$ solution.

3.4. Adsorption Consideration. Basic parameters which are descriptors of the nature and modes of adsorption of organic inhibitor on the corroding metal surface can be provided by adsorption isotherms which depend on the degree of surface coverage, $\theta$. The observed inhibition of the corrosion of mild steel in $1.0 \mathrm{M} \mathrm{H}_{2} \mathrm{SO}_{4}$ solution indicates high degree of surface coverage. From a theoretical perspective, the adsorption route is regarded as a substitution process between the organic inhibitor in the aqueous solution $\left(\mathrm{Inh}_{\text {sol }}\right)$ and water 


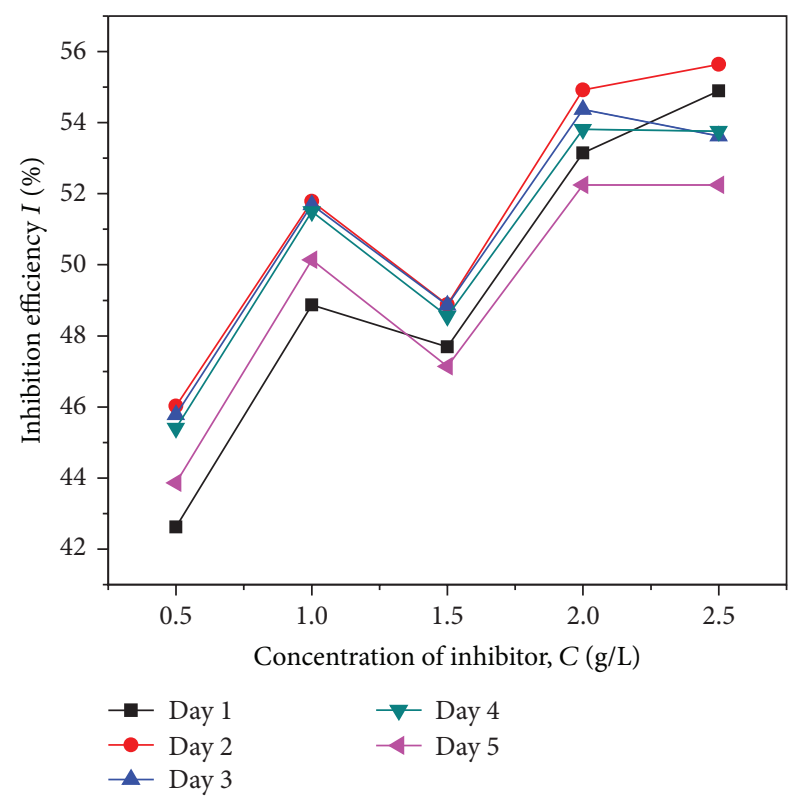

FIGURE 1: Variation of inhibition efficiency with concentration of EHEC.

molecules adsorbed at the metal surface $\left(\mathrm{H}_{2} \mathrm{O}_{\text {ads }}\right)$ as follows [36-38]:

$$
\mathrm{Inh}_{(\mathrm{sol})}+x \mathrm{H}_{2} \mathrm{O}_{(\mathrm{ads})} \Longleftrightarrow \mathrm{Inh}_{(\mathrm{ads})}+x \mathrm{H}_{2} \mathrm{O}_{(\mathrm{sol})},
$$

where $x$ represents the number of water molecules replaced by one molecule of adsorbed inhibitor. The adsorption bond strength is dependent on the composition of the metal and corrodent, inhibitor structure, concentration, and orientation, as well as temperature. Since EHEC can be protonated in the presence of strong acids, it is quite necessary to consider both cationic and molecular species when discussing the adsorption process of EHEC. Figure 3 shows the plot of $C / \theta$ versus $C$ to be linear, which is in agreement with the Langmuir equation [39]:

$$
\frac{C}{\theta}=\frac{n}{K_{\mathrm{ads}}}+n C,
$$

where $C$ is the concentration of inhibitor and $K_{\text {ads }}$ is the equilibrium constant for the adsorption-desorption process.

In general, $K_{\text {ads }}$ represents the adsorption power of the inhibitor molecule on the metal surface. The positive values confirm the adsorbability of EHEC on the metal surface. The linear plots obtained in Figure 3 suggest that EHEC adsorption from $1.0 \mathrm{M} \mathrm{H}_{2} \mathrm{SO}_{4}$ solution followed the Langmuir isotherm, though the isotherm parameters indicate some deviations from ideal Langmuir behaviour. The slope deviates from unity (see $n$ values in Table 3 ) with nonzero intercept on the $y$-axis, which could be traced to some limitations in the underlying assumptions. The results in fact imply that each EHEC molecule occupies $n$ active corrosion sites on the mild steel surface in $1.0 \mathrm{M} \mathrm{H}_{2} \mathrm{SO}_{4}$ solution.
The free energy of adsorption $\left(\Delta G_{\text {ads }}\right)$ obtained from (7) which is evaluated from $K_{\text {ads }}$ obtained from intercepts of the Langmuir plots is given in Table 3:

$$
\Delta G_{\mathrm{ads}}=-R T \ln \left(55.5 K_{\mathrm{ads}}\right) \text {, }
$$

where $R$ and $T$ are the universal gas constant and absolute temperature, respectively. The other parameter retains its previous meaning. The large negative $\Delta G_{\text {ads }}$ values implied that the adsorption of EHEC on the mild steel surface was favourable from thermodynamics point of view and indicated that the inhibitor was strongly adsorbed, covering both anodic and cathodic regions.

In addition, it is important to note that adsorption free energy values of $-20 \mathrm{~kJ} \mathrm{~mol}^{-1}$ or less negative are associated with an electrostatic interaction between charged molecules and charged metal surface (physical adsorption). On the other hand, adsorption free energy values of $-40 \mathrm{Kj} \mathrm{mol}^{-1}$ or more negative values involve charge sharing or transfer from the inhibitor molecules to the metal surface to form a coordinate covalent bond (chemical adsorption) [40].

3.5. Impedance Measurements. Electrochemical impedance spectroscopy analyses provide insight into the kinetics of electrode processes as well as the surface characteristics of the electrochemical system of interest. Figure 4 presents the impedance spectra measured at $E_{\text {corr }}$ after 30 minutes of immersion and exemplified the Nyquist plots obtained for mild steel in $1.0 \mathrm{M} \mathrm{H}_{2} \mathrm{SO}_{4}$ solution in the absence and presence of EHEC and EHEC + KI. The observed increase in the impedance parameters in inhibited solutions is associated with the corrosion inhibiting effect of EHEC. The Nyquist plots for all systems generally have the form of only one depressed semicircle, corresponding to one time constant, although a slight sign of low-frequency inductive behaviour can be discerned. The depression of the capacitance semicircle with centre below the real axis suggests a distribution of the capacitance due to inhomogeneities associated with the electrode surface.

The presence of a single time constant may be attributed to the short exposure time in the corrosive medium which is not adequate to reveal degradation of the substrate [41]. A polarization resistance $\left(R_{p}\right)$ can be extracted from the intercept of the low-frequency loop at the real axis of impedance $\left(Z_{\mathrm{re}}\right)$ in the Nyquist plots, since the inductive loop is negligible. The value of $R_{p}$ is very close to that of the charge transfer resistance $R_{\mathrm{ct}}$, which can be extracted from the diameter of the semicircle $[41,42]$. The impedance spectra for the Nyquist plots were thus adequately analyzed by being fit to the equivalent circuit model $R_{s}\left(Q_{\mathrm{dl}} R_{\mathrm{ct}}\right)$, which has been previously used to model the mild steel/acid solution interface $[41,43]$.

The values of the impedance parameters derived from the Nyquist plots using the selected equivalent circuit model $R_{s}\left(Q_{\mathrm{dl}} R_{\mathrm{ct}}\right)$ are given in Table 4. The terms $Q_{\mathrm{dl}}$ and $n$, respectively, represent the magnitude and exponent of the constant phase element (CPE) of the double layer. The CPE, with impedance given by $Z_{\mathrm{CPE}}=Q^{-1}(j w)^{-n}$, where $j$ is an imaginary number and $w$ is the angular frequency in 


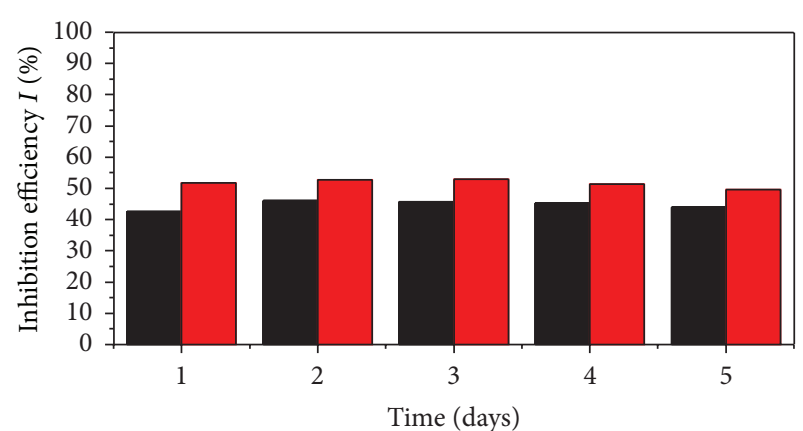

- $0.5 \mathrm{~g} / \mathrm{L}$ EHEC

$\square 0.5 \mathrm{~g} / \mathrm{L}$ EHEC $+\mathrm{KI}$

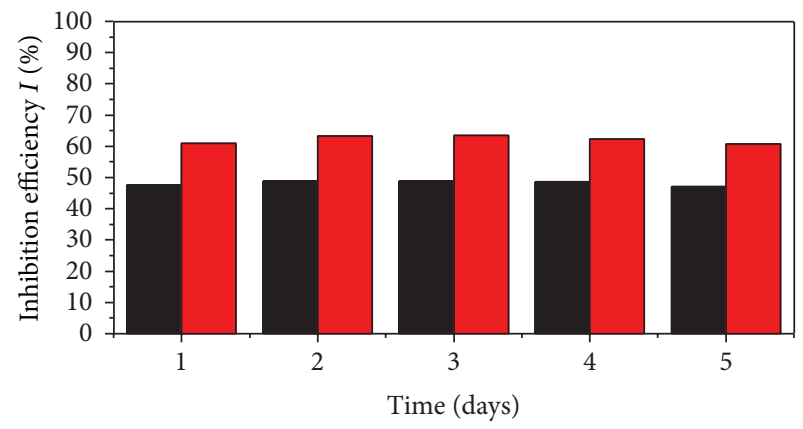

- $1.5 \mathrm{~g} / \mathrm{L}$ EHEC

$\square 1.5 \mathrm{~g} / \mathrm{L}$ EHEC + KI

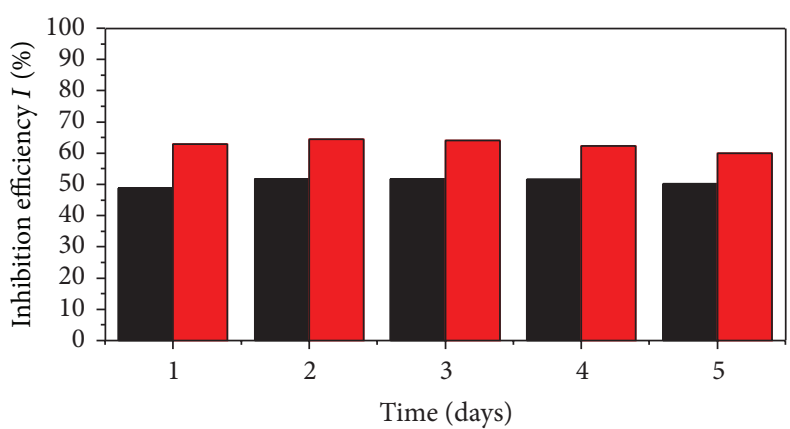

$1.0 \mathrm{~g} / \mathrm{L} \mathrm{EHEC}$

- $1.0 \mathrm{~g} / \mathrm{L} \mathrm{EHEC}+\mathrm{KI}$

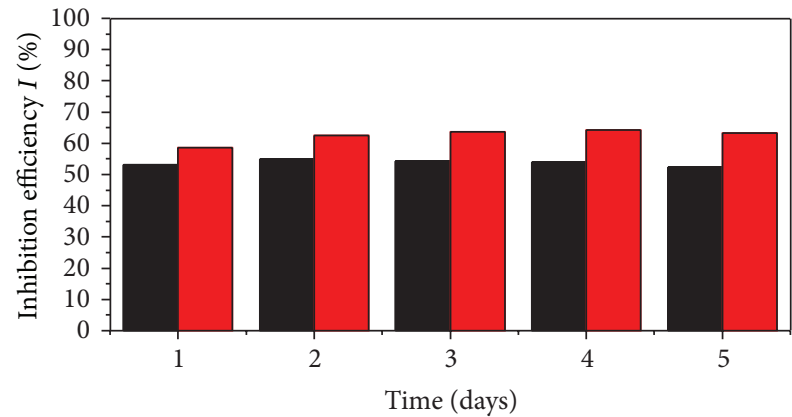

$2.0 \mathrm{~g} / \mathrm{L}$ EHEC

$\square 2.0 \mathrm{~g} / \mathrm{L} \mathrm{EHEC}+\mathrm{KI}$

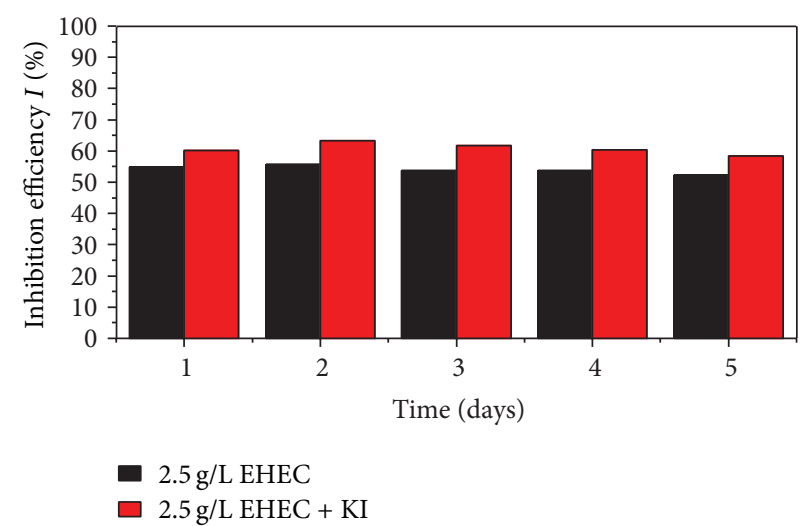

FIGURE 2: Synergistic effect between KI and EHEC on the variation of inhibition efficiency with time.

$\mathrm{rad} / \mathrm{s}$ is used in place of a capacitor to compensate for the deviations from ideal dielectric behaviour associated with the nonhomogeneity of the electrode surface. Introduction of EHEC into the acid corrodent leads to an increase in $R_{\mathrm{ct}}$ and a reduction of $Q_{\mathrm{dl}}$, indicating a hindering of the corrosion reaction. The decrease in $Q_{\mathrm{dl}}$ values, which normally results from a decrease in the dielectric constant and/or an increase in the double layer thickness, is due to inhibitor adsorption on the metal/electrolyte interface [44]. This implies that EHEC reduces the corrosion rate of the mild steel specimen by virtue of adsorption on the metal/electrolyte interface, a fact that has been previously established. A quantitative measure of the protective effect can be obtained by comparing the values of the charge transfer resistance in the absence $\left(R_{\mathrm{ct}}\right)$ and presence of inhibitor $\left(R_{\text {ctinh }}\right)$ as follows:

$$
I \%=\left[1-\frac{R_{\mathrm{ct}}}{R_{\mathrm{ctinh}}}\right] \times 100,
$$

where $\left(R_{\mathrm{ctinh}}\right)$ and $R_{\mathrm{ct}}$ are the charge transfer resistance for inhibited and uninhibited systems, respectively.

The double layer capacitance values of the systems were also examined and calculated using the expression:

$$
C_{\mathrm{dl}}=\frac{1}{2 \pi f_{\max } R_{\mathrm{ct}}},
$$

where $f_{\max }$ is the maximum frequency. The obtained values of $C_{\mathrm{dl}}$ are presented in Table 4. 


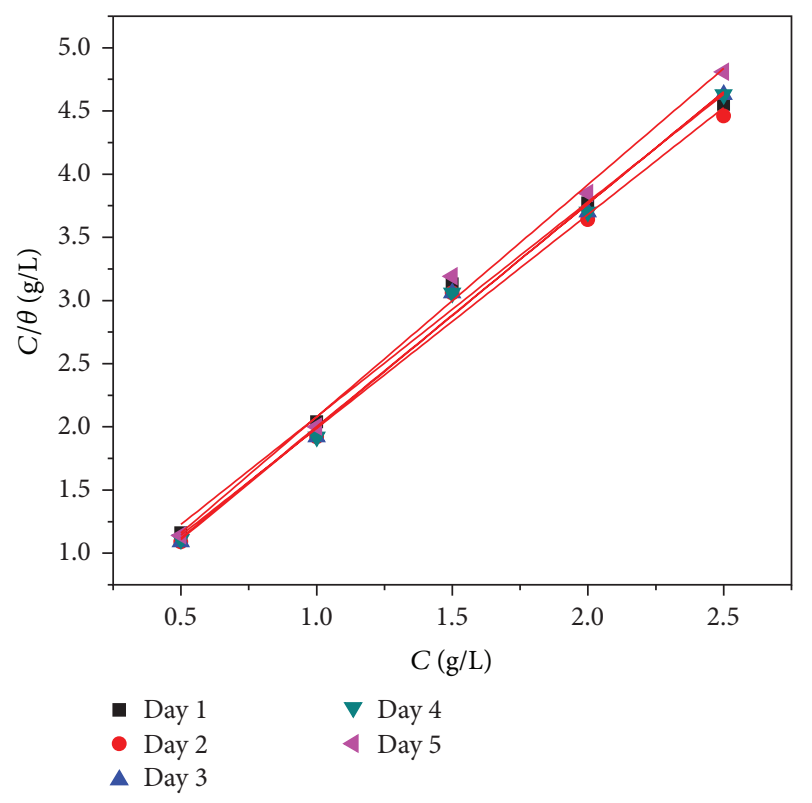

Figure 3: Langmuir isotherm for EHEC adsorption on mild steel surface in $1.0 \mathrm{M} \mathrm{H}_{2} \mathrm{SO}_{4}$.

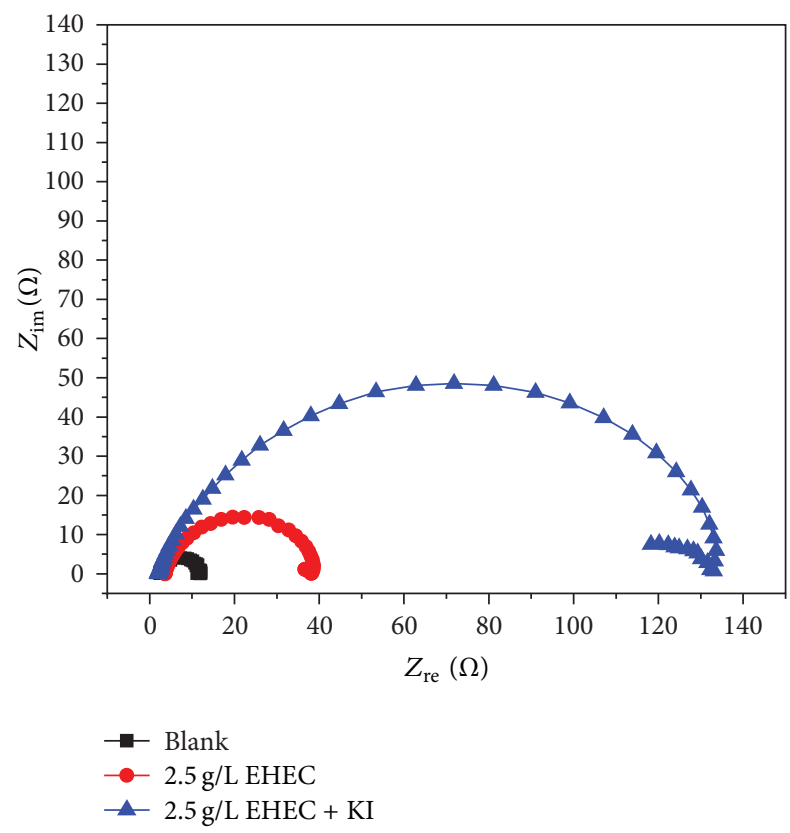

FIGURE 4: Nyquist impedance spectra of mild steel corrosion in 1.0 $\mathrm{M} \mathrm{H}_{2} \mathrm{SO}_{4}$ in the absence and presence of EHEC and EHEC + KI.

Lower double layer capacitance suggests reduced electric charge stored, which is a consequence of increased adsorption layer that acted as a dielectric constant. The increase in $R_{\mathrm{ct}}$ values in inhibited systems which corresponds to an increase in the diameter of the Nyquist semicircle confirms that the corrosion inhibiting effect of EHEC and EHEC + KI and is much more pronounced in the latter system, implying that KI synergistically enhanced the corrosion inhibiting effect of EHEC. In other words, lower $C_{\mathrm{dl}}$ values correspond
TABLE 3: Adsorption parameters from modified Langmuir isotherm.

\begin{tabular}{lcccc}
\hline Day & $R^{2}$ & $n$ & $K_{\text {ads }}$ & $\Delta G_{\text {ads }}\left(\mathrm{kJ} \mathrm{mol}^{-1}\right)$ \\
\hline 1 & 0.990 & 1.702 & 4.515 & -45.381 \\
2 & 0.988 & 1.692 & 5.795 & -58.246 \\
3 & 0.993 & 1.772 & 7.982 & -80.228 \\
4 & 0.993 & 1.764 & 7.412 & -74.498 \\
5 & 0.992 & 1.834 & 7.627 & -76.659 \\
\hline
\end{tabular}

to reduced double layer capacitance, which, according to the Helmholtz model (10), results from a decrease in the dielectric constant $(\varepsilon)$ or an increase in the interfacial layer thickness $(\delta)$ :

$$
C_{\mathrm{dl}}=\frac{\varepsilon \varepsilon_{o} A}{\delta}
$$

where $\varepsilon$ is the dielectric constant of the medium, $\varepsilon_{o}$ is the vacuum permittivity, $A$ is the electrode area, and $\delta$ is the thickness of the interfacial layer.

Since adsorption of an organic inhibitor on a metal surface involves the replacement of adsorbed water molecules on the surface, the smaller dielectric constant of the organic molecule compared to water as well as the increased thickness of interfacial layer due to inhibitor adsorption acted simultaneously to reduce the double layer capacitance. This provides experimental evidence of adsorption of EHEC on mild steel surface. The significantly lower $C_{\mathrm{dl}}$ value of the EHEC + KI system supports the assertion that the iodide ion significantly enhances adsorption of EHEC on the metal/solution interface.

3.6. Polarization Measurements. Figure 5 shows the polarization curves of mild steel dissolution in $1.0 \mathrm{M} \mathrm{H}_{2} \mathrm{SO}_{4}$ solution in the absence and presence of EHEC and EHEC + KI. Introduction of EHEC and EHEC + KI into the acid solution was observed to shift the corrosion potentials of both inhibited systems slightly in the negative direction and in both cases inhibited the anodic metal dissolution reaction as well as the cathodic hydrogen evolution reaction. Since the $E_{\text {corr }}$ is not altered significantly, the implication is that the corrosion inhibition process is under mixed control with predominant cathodic effect. In addition, following observations in Figure 5, both cathodic and anodic partial reactions are affected as evident in decrease in the corrosion current densities. This implies that EHEC functioned as a mixedtype inhibitor for both systems. However, marked cathodic partial hydrogen evolution reaction is discerned. Moreso, documented report [45] has it that when displacement in $E_{\text {corr }}$ is $>85 \mathrm{mV}$, the inhibitor can be regarded as a cathodic or anodic type inhibitor and if the displacement in $E_{\text {corr }}$ is $<85 \mathrm{mV}$, the inhibitor can be seen as mixed type. In the present study, the displacement in the corrosion potential $\left(E_{\text {corr }}\right)$ in the presence of EHEC and EHEC + KI shifted 9.52 and $20.75 \mathrm{mV}$, respectively, in the cathodic direction, compared to the blank, which is a confirmation that the inhibitor acts as a mixed-type inhibitor with predominant cathodic effect. 
TABLE 4: Impedance and polarization parameters for mild steel in $0.5 \mathrm{M} \mathrm{H}_{2} \mathrm{SO}_{4}$ in the presence and absence of EHEC and EHEC + KI.

\begin{tabular}{|c|c|c|c|c|c|c|c|}
\hline \multirow{2}{*}{ System } & \multicolumn{4}{|c|}{ Impedance data } & \multicolumn{3}{|c|}{ Polarization data } \\
\hline & $R_{\mathrm{ct}}$ & $n$ & I.E. $\%$ & $C_{\mathrm{dl}}\left(\mathrm{uF} \mathrm{cm}^{-2}\right) \times 10^{-3}$ & $E_{\text {corr }}(\mathrm{mV}(\mathrm{SCE}))$ & $R_{p}\left(\Omega \mathrm{cm}^{2}\right)$ & I.E. $\%$ \\
\hline Blank & 11.931 & 0.889 & - & 14.65 & -468.35 & 16.27 & - \\
\hline EHEC & 37.502 & 0.876 & 68.19 & 5.13 & -477.87 & 58.04 & 71.97 \\
\hline $\mathrm{EHEC}+\mathrm{KI}$ & 133.37 & 0.854 & 91.05 & 2.41 & -489.10 & 177.51 & 90.83 \\
\hline
\end{tabular}

TABLE 5: Calculated values of quantum chemical properties for the most stable conformations of EHEC.

\begin{tabular}{lc}
\hline Property & EHEC \\
\hline$E_{\text {HOMO }}(\mathrm{eV})$ & -6.154 \\
$E_{\mathrm{LUMO}}(\mathrm{eV})$ & -2.323 \\
$E_{\mathrm{LUMO}-\mathrm{HOMO}}(\mathrm{eV})$ & 3.831 \\
Maximum $f^{+}($Mulliken $)$ & $0.015 \mathrm{O}(12)$ \\
Maximum $f^{-}$(Mulliken) & $0.165 \mathrm{O}(12)$ \\
\hline
\end{tabular}

Inhibition efficiency was calculated from the polarization data as follows:

$$
I \%=\left[1-\frac{R_{p}}{R_{p}^{\text {inh }}}\right] \times 100
$$

where $R_{p}$ and $R_{p}{ }^{\text {inh }}$ are polarization resistance for uninhibited and inhibited systems, respectively. The calculated values are given in Table 4 . Observations from Table 4 show that the inhibition efficiencies obtained from both impedance and polarization results are comparable. The data confirm the consistency of EHEC and EHEC $+\mathrm{KI}$ at the prevailing experimental condition.

The cooperative effect between EHEC and KI in hindering the corrosion of mild steel in $1.0 \mathrm{M} \mathrm{H}_{2} \mathrm{SO}_{4}$ solution is also evident in both the Nyquist and Tafel polarization plots. Addition of KI resulted in a significant increase in the diameter of the Nyquist semicircle and hence an increase in $R_{\mathrm{ct}}$ as well as $I \%$ and a decrease in the corrosion current density of the Tafel polarization curves. The presence of iodide ions shifts $E_{\text {corr }}$ more in the cathodic direction and further decreases the anodic and cathodic reaction kinetics. The mechanism of this synergistic effect has been described in detail in some reports [46]. The iodide ions are strongly chemisorbed on the corroding mild steel surface and facilitate EHEC adsorption by acting as intermediate bridges between the positively charged metal surface and EHEC cations. This stabilizes the adsorption of EHEC on the mild steel surface, leading to higher surface coverage. To account for the above observations, it is necessary to recognize that the process of adsorption of an organic inhibitor on a corroding metal surface depends on factors such as the nature and surface charge on the metal in the corrosive medium as well as the inhibitor structure. Consequently, more iodide ions are adsorbed on mild steel which presents a more positive surface, giving rise to increased synergistic interactions with protonated EHEC species and hence higher inhibition efficiencies.

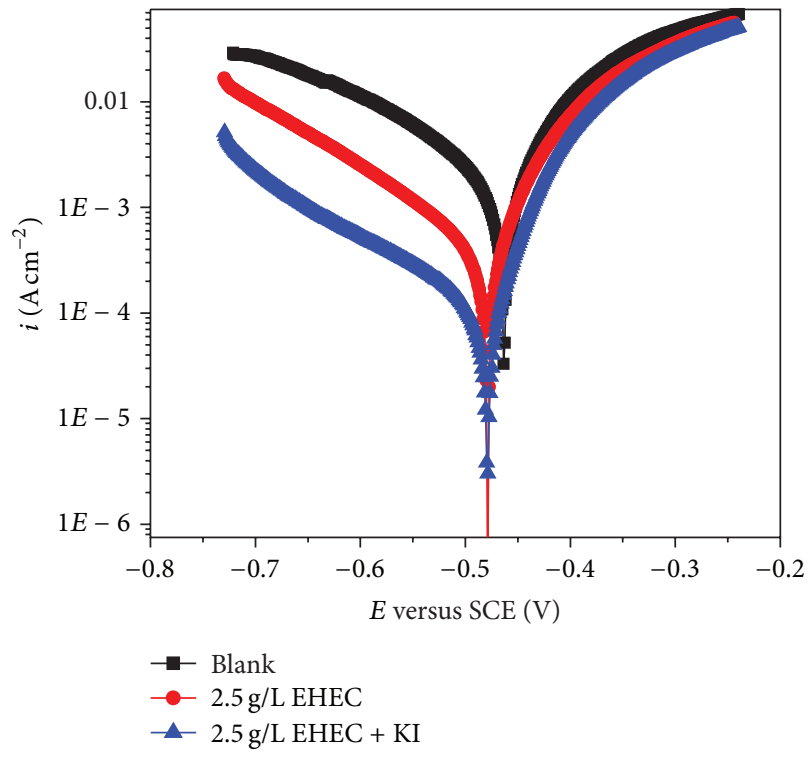

Figure 5: Polarization curves of mild steel corrosion in $1.0 \mathrm{M} \mathrm{H}_{2} \mathrm{SO}_{4}$ in the absence and presence of EHEC and EHEC + KI.

3.7. Quantum Chemical Calculations. The inhibition effectiveness of inhibitors has been reported to correlate with the quantum chemical parameters such as HOMO (the highest occupied molecular orbital), LUMO (the lowest unoccupied molecular orbital), and the energy gap between the LUMO and $\operatorname{HOMO}\left(\Delta E=E_{\mathrm{LUMO}}-E_{\mathrm{HOMO}}\right)$ [47-49]. A high $E_{\text {HOMO }}$ (less negative) is associated with the capacity of a molecule to donate electrons to an appropriated acceptor with empty molecular orbital that facilitated the adsorption process and therefore indicated good performance of the corrosion inhibitor [50]. $E_{\mathrm{LUMO}}$ corresponds to a tendency for electron acceptance. Based on this, the calculated difference, $\Delta E$, demonstrates inherent electron donating ability and measures the interaction of the inhibitor molecule with the metal surface.

According to the frontier molecular orbital theory of chemical reactivity, transition of electrons is due to an interaction between the frontier orbitals, HOMO and LUMO, of reacting species. The energy of $\mathrm{HOMO}$ is directly related to the ionization potential and characterizes the susceptibility of the molecule toward attack by electrophiles. The energy of LUMO is directly related to the electron affinity and characterizes the susceptibility of the molecule toward attack by nucleophile. The lower the values of $E_{\mathrm{LUMO}}$, the stronger the electron accepting ability of the molecule. 


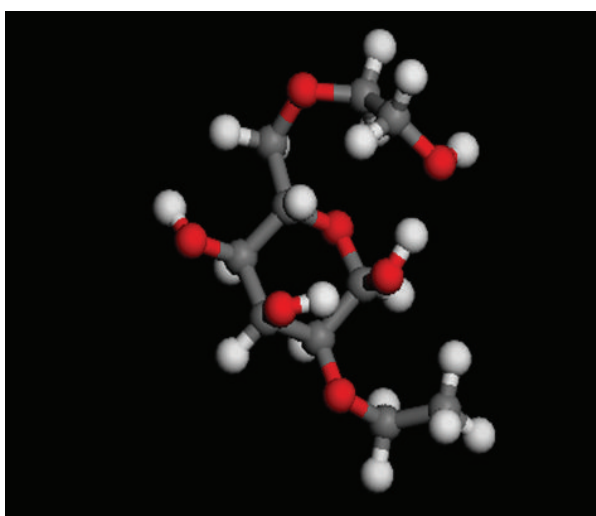

(a) Optimized structure

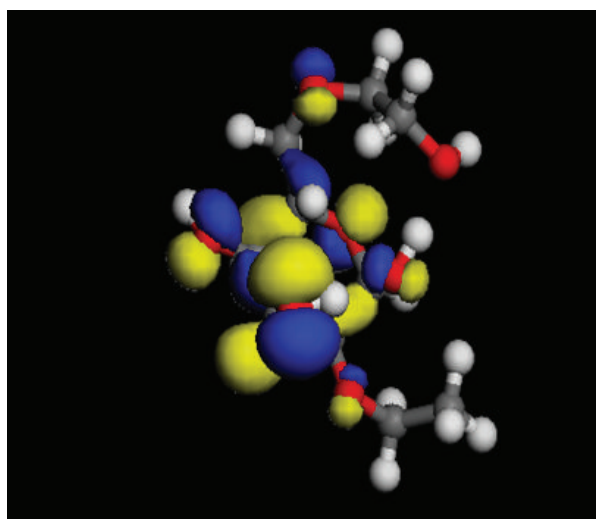

(c) HOMO orbital

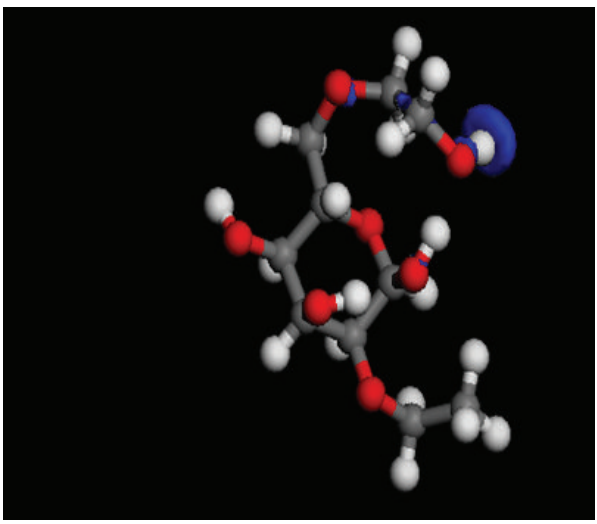

(e) Fukui function for nucleophilic attack

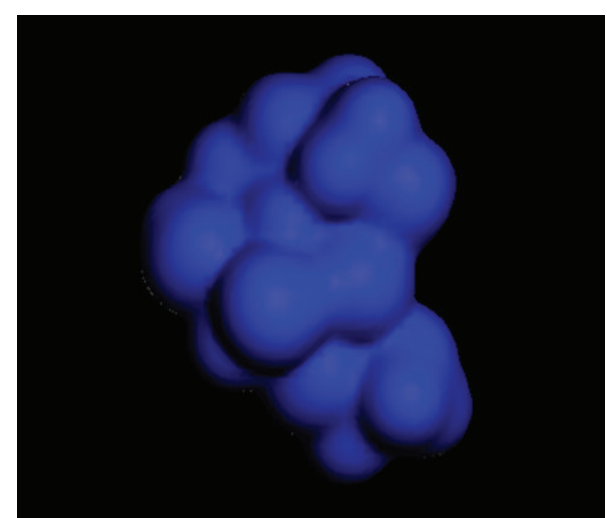

(b) Total electron density

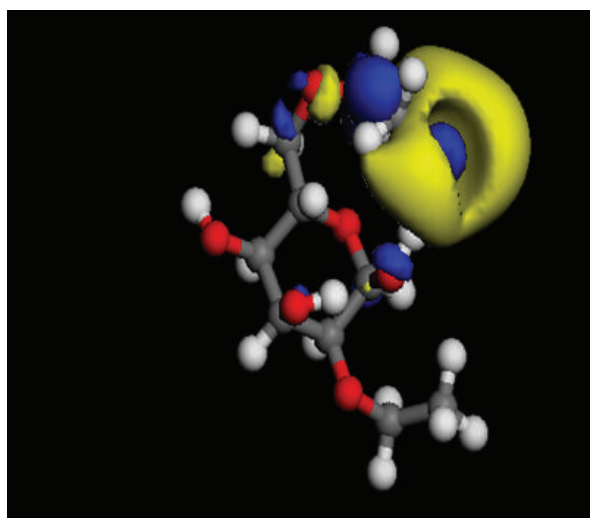

(d) LUMO orbital

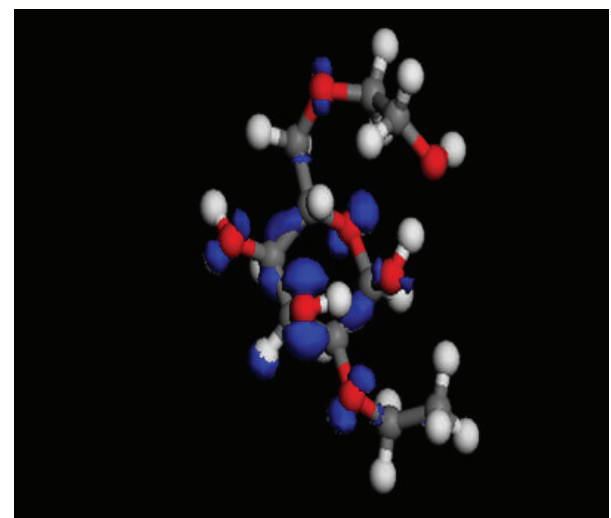

(f) Fukui function for electrophilic attack

FIGURE 6: Electronic properties of ethyl hydroxyethyl cellulose (EHEC) [C, grey; H, white; O, red].

The electronic structure of EHEC, the distribution of frontier molecular orbital, and Fukui indices have been modeled in order to establish the active sites as well as local reactivity of the inhibiting molecules. This was achieved using the DFT electronic structure programs, Forcite and DMol3, and using a Mulliken population analysis. Electronic parameters for the simulation include restricted spin polarization using the DND basis set as the Perdew Wang (PW) local correlation density functional. The geometry optimized structures of EHEC, HOMO and LUMO orbitals, Fukui functions, and the total electron density are presented in Figure 6. In the EHEC molecule, the HOMO orbital is saturated around the aromatic nucleus which is the region of highest electron density and often the site at which electrophiles attack, and represents the active centres, with the utmost ability to bond to the metal surface. The LUMO orbital is saturated around the ethoxy function and represents the site at which nucleophilic attack occurs.

Local reactivity was analyzed by means of the Fukui indices to assess the active regions in terms of nucleophilic and electrophilic behaviour. Thus, the site for nucleophilic attack will be the place where the value of $f^{+}$is maximum. In 
turn, the site for electrophilic attack is controlled by the value of $f^{-}$. The values of $E_{\mathrm{HOMO}}, E_{\mathrm{LUMO}}, \Delta E$, and Fukui functions are given in Table 5. Higher values of $E_{\mathrm{HOMO}}$ indicate a greater disposition of a molecule to donate electrons to a metal surface. In the same way, low values of the energy of the gap, $\Delta E$ will afford good inhibition efficiency, since the energy required to remove an electron from the last occupied orbital will be minimized [51]. The above descriptors, however, suggest that EHEC possessed good inhibiting potential. This is in agreement with the experimental findings.

\section{Conclusions}

Ethyl hydroxyethyl cellulose was found to be an effective inhibitor of mild steel in $1.0 \mathrm{M} \mathrm{H}_{2} \mathrm{SO}_{4}$ solution and its inhibition efficiency increased with increasing concentration. The corrosion process is inhibited by adsorption of EHEC on the mild steel surface following the modified Langmuir isotherm. The inhibiting action is attributed to general adsorption of both protonated and molecular species of the additive on the cathodic and anodic sites on the corroding mild steel surface. In addition, corrosion inhibition is due to the formation of a chemisorbed film on the mild steel surface. The EIS measurement confirmed the adsorption of EHEC and EHEC $+\mathrm{KI}$ on the mild steel surface. Polarization studies showed that EHEC and EHEC + KI were mixed-type inhibitor systems with predominant cathodic effect. The theoretical study demonstrated that the inhibition efficiency is related to molecular structure of inhibitor whereby increase in $E_{\mathrm{HOMO}}$ and decrease in $E_{\mathrm{LUMO}}$ favoured inhibition efficiency.

\section{Conflict of Interests}

The authors hereby declare that there is no conflict of interests regarding the publication of this paper.

\section{Acknowledgments}

The authors are grateful to Udemmadu Tochukwu and Ochiogu Valentine for assistance in carrying out some measurements.

\section{References}

[1] E. E. Oguzie, Y. Li, and F. H. Wang, "Effect of ascorbic acid on mild steel dissolution in sulphuric acid solution investigated by electrochemical polarization and surface probe techniques," Journal of Applied Electrochemistry, vol. 37, no. 10, pp. 1183-1190, 2007.

[2] L. G. da Trindade and R. S. Goncalves, "Evidence of caffeine adsorption on a low-carbon steel surface in ethanol," Corrosion Science, vol. 51, no. 8, pp. 1578-1583, 2009.

[3] F. Bentiss, M. Outirite, M. Traisnel et al., "Improvement of corrosion resistance of carbon steel in hydrochloric acid medium by 3,6-bis(3-pyridyl)pyridazine," International Journal of Electrochemical Science, vol. 7, no. 2, pp. 1699-1723, 2012.

[4] S. M. A. Shibli and V. S. Saji, "Co-inhibition characteristics of sodium tungstate with potassium iodate on mild steel corrosion," Corrosion Science, vol. 47, no. 9, pp. 2213-2224, 2005.
[5] E. E. Oguzie, C. Unaegbu, C. N. Ogukwe, B. N. Okolue, and A. I. Onuchukwu, "Inhibition of mild steel corrosion in sulphuric acid using indigo dye and synergistic halide additives," Materials Chemistry and Physics, vol. 84, no. 2-3, pp. 363-368, 2004.

[6] V. Kumpawat, U. Garg, and R. K. Tak, "Corrosion inhibition of aluminium in acid media by naturally occurring plant Artocarpus heterophyllus and Acacia senegal," Journal of Indian Council of Chemists, vol. 26, no. 1, pp. 82-84, 2009.

[7] I.A. Akpan and N. O. Offiong, "Effect of ethanolamine and ethylamine on the entropy content of the corrosion of mild steel in tetraoxosulphate (VI) acid solution," Chemistry and Materials Research, vol. 2, no. 7, pp. 40-47, 2012.

[8] M. Kissi, M. Bouklah, B. Hammouti, and M. Benkaddour, "Establishment of equivalent circuits from electrochemical impedance spectroscopy study of corrosion inhibition of steel by pyrazine in sulphuric acidic solution," Applied Surface Science, vol. 252, no. 12, pp. 4190-4197, 2006.

[9] S. Rajendran, S. P. Sridevi, N. Anthony, A. J. Amalraj, and M. Sundaravadivelu, "Corrosion behaviour of carbon steel in polyvinyl alcohol," Anti-Corrosion Methods and Materials, vol. 52, no. 2, pp. 102-107, 2005.

[10] B. Qian, J. Wang, M. Zheng, and B. Hou, "Synergistic effect of polyaspartic acid and iodide ion on corrosion inhibition of mild steel in $\mathrm{H}_{2} \mathrm{SO}_{4}$," Corrosion Science, vol. 75, pp. 184-192, 2013.

[11] D. A. Arthur, A. Jonathan, P. O. Ameh, and C. Anya, "A review on the assessment of polymeric materials used as corrosion inhibitor of metals and alloys," International Journal of Industrial Chemistry, vol. 4, article 2, pp. 1-9, 2013.

[12] S. A. Umoren, Y. Li, and F. H. Wang, "Effect of polyacrylic acid on the corrosion behaviour of aluminium in sulphuric acid solution," Journal of Solid State Electrochemistry, vol. 14, no. 12, pp. 2293-2305, 2010.

[13] S. Banerjee, A. Mishra, M. M. Singh, B. Maiti, B. Ray, and P. Maiti, "Highly efficient polyurethane ionomer corrosion inhibitor: the effect of chain structure," RSC Advances, vol. 1, no. 2, pp. 199-210, 2011.

[14] A. K. Dubey and G. Singh, "Corrosion inhibition of mild steel in sulphuric acid solution by using polyethylene glycol methyl ether (PEGME)," Portugaliae Electrochimica Acta, vol. 25, no. 2, pp. 221-235, 2007.

[15] E. E. Oguzie, S. G. Wang, Y. Li, and F. H. Wang, "Influence of iron microstructure on corrosion inhibitor performance in acidic media," The Journal of Physical Chemistry C, vol. 113, no. 19, pp. 8420-8429, 2009.

[16] S. A. Umoren, E. E. Ebenso, P. C. Okafor, and O. Ogbobe, "Water-soluble polymers as corrosion inhibitors," Pigment \& Resin Technology, vol. 35, no. 6, pp. 346-352, 2006.

[17] S. A. Umoren and I. B. Obot, "Polyvinylpyrollidone and polyacrylamide as corrosion inhibitors for mild steel in acidic medium," Surface Review and Letters, vol. 15, no. 3, pp. 277-284, 2008.

[18] H. Ashassi-Sorkhabi and N. Ghalebsaz-Jeddi, "Inhibition effect of polyethylene glycol on the corrosion of carbon steel in sulphuric acid," Materials Chemistry and Physics, vol. 92, no. 2-3, pp. 480-486, 2005.

[19] A. Yurt, V. Butun, and B. Duran, "Effect of the molecular weight and structure of some novel water-soluble triblock copolymers on the electrochemical behaviour of mild steel," Materials Chemistry and Physics, vol. 105, no. 1, pp. 114-121, 2007. 
[20] S. A. Umoren, "Polymers as corrosion inhibitors for metals in different media-a review," The Open Corrosion Journal, vol. 2, pp. 175-188, 2009.

[21] K. F. Khaled, "Corrosion control of copper in nitric acid solutions using some amino acids-a combined experimental and theoretical study," Corrosion Science, vol. 52, no. 10, pp. 3225-3234, 2010.

[22] E. E. Oguzie, C. O. Akalezi, C. K. Enenebeaku, and J. N. Aneke, "Corrosion inhibition and adsorption behavior of malachite green dye on aluminum corrosion," Chemical Engineering Communications, vol. 198, no. 1, pp. 46-60, 2011.

[23] J. Cruz, T. Pandiyan, and E. Garcia-Ochoa, "A new inhibitor for mild carbon steel: electrochemical and DFT studies," Journal of Electroanalytical Chemistry, vol. 583, no. 1, pp. 8-16, 2005.

[24] J. Cruz, R. Martinez, J. Genesca, and E. Garcia-Ochoa, "Experimental and theoretical study of 1-(2-ethylamino)-2methylimidazoline as an inhibitor of carbon steel corrosion in acid media," Journal of Electroanalytical Chemistry, vol. 566, no. 1, pp. 111-121, 2004.

[25] E. E. Oguzie, Y. Li, S. G. Wang, and F. Wang, "Understanding corrosion inhibition mechanisms-experimental and theoretical approach," RSC Advances, vol. 1, no. 5, pp. 866-873, 2011.

[26] A. Kumar, A. Sankara, M. Kumaravel, and S. Rameshkumar, "Clitoria ternatea-extracts as corrosion inhibitor for mild steel in acid medium," International Journal of Engineering Research and Development, vol. 8, no. 5, pp. 64-67, 2013.

[27] E. E. Oguzie, G. N. Onuoha, and A. I. Onuchukwu, "Inhibitory mechanism of mild steel corrosion in $2 \mathrm{M}$ sulphuric acid solution by methylene blue dye," Materials Chemistry and Physics, vol. 89, no. 2-3, pp. 305-311, 2005.

[28] E. E. Oguzie, C. K. Enenebeaku, C. O. Akalezi, S. C. Okoro, A. A. Ayuk, and E. N. Ejike, "Adsorption and corrosion-inhibiting effect of Dacryodis edulis extract on low-carbon-steel corrosion in acidic media," Journal of Colloid and Interface Science, vol. 349, no. 1, pp. 283-292, 2010.

[29] E. E. Oguzie, "Corrosion inhibition of aluminium in acidic and alkaline media by Sansevieria trifasciata extract," Corrosion Science, vol. 49, no. 3, pp. 1527-1539, 2007.

[30] S. A. Umoren, I. B. Obot, and E. E. Ebenso, "Corrosion inhibition of aluminium using exudate gum from Pachylobus edulis in the presence of halide ions in HCl," E-Journal of Chemistry, vol. 5, no. 2, pp. 355-364, 2008.

[31] P. C. Okafor, E. E. Ebenso, and U. J. Ekpe, "Azadirachta indica extracts as corrosion inhibitor for mild steel in acid medium," International Journal of Electrochemical Science, vol. 5, no. 7, pp. 978-993, 2010.

[32] M. Lebrini, F. Robert, A. Lecente, and C. Roos, "Corrosion inhibition of C38 steel in $1 \mathrm{M}$ hydrochloric acid medium by alkaloids extract from Oxandra asbeckii plant "' Corrosion Science, vol. 53, no. 2, pp. 687-695, 2011.

[33] E. E. Oguzie, "Influence of halide ions on the inhibitive effect of congo red dye on the corrosion of mild steel in sulphuric acid solution," Materials Chemistry and Physics, vol. 87, no. 1, pp. 212217, 2004.

[34] E. E. Oguzie, "Inhibition of acid corrosion of mild steel by Telfaria occidentalis extract," Pigment \& Resin Technology, vol. 34, no. 6, pp. 321-326, 2005.

[35] E. E. Oguzie, V. O. Njoku, C. K. Enenebeaku, C. O. Akalezi, and C. Obi, "Effect of hexamethylpararosaniline chloride (crystal violet) on mild steel corrosion in acidic media," Corrosion Science, vol. 50, no. 12, pp. 3480-3486, 2008.
[36] M. A. Ameer, E. Khamis, and G. al-Senani, "Effect of temperature on stability of adsorbed inhibitors on steel in phosphoric acid solution," Journal of Applied Electrochemistry, vol. 32, no. 2, pp. 149-156, 2002.

[37] P. C. Okafor, E. E. Oguzie, G. E. Iniama, M. E. Ikpi, and U. J. Ekpe, "Corrosion inhibition properties of thiosemicarbazone and semicarbazone derivatives in concentrated acid environment," Global Journal of Pure and Applied Sciences, vol. 14, no. 1, pp. 89-96, 2008.

[38] I. B. Obot, "Synergistic effect of nizoral and iodide ions on the corrosion inhibition of mild steel in sulphuric acid solution," Portugaliae Electrochimica Acta, vol. 27, no. 5, pp. 539-553, 2009.

[39] N. O. Eddy, S. A. Odoemelam, and A. O. Odiongenyi, "Ethanol extract of musa species peels as a green corrosion inhibitor for mild steel: kinetics, adsorption and thermodynamic considerations," Electronic Journal of Environmental, Agricultural and Food Chemistry, vol. 8, no. 4, pp. 243-255, 2009.

[40] M. H. Hussin and M. J. Kassim, "The corrosion inhibition and adsorption behavior of Uncaria gambir extract on mild steel in $1 \mathrm{M} \mathrm{HCl}, "$ Materials Chemistry and Physics, vol. 125, no. 3, pp. 461-468, 2010.

[41] V. K. W. Grips, V. E. Selvi, H. C. Barshilia, and K. S. Rajam, "Effect of electroless nickel interlayer on the electrochemical behavior of single layer $\mathrm{CrN}$, TiN, TiAlN coatings and nanolayered TiAlN/CrN multilayer coatings prepared by reactive dc magnetron sputtering," Electrochimica Acta, vol. 51, no. 17, pp. 3461-3468, 2006.

[42] E. E. Oguzie, "Studies on the inhibitive effect of Occimum viridis extract on the acid corrosion of mild steel," Materials Chemistry and Physics, vol. 99, no. 2-3, pp. 441-446, 2006.

[43] B. Wang, M. Du, J. Zhang, and C. J. Gao, "Electrochemical and surface analysis studies on corrosion inhibition of Q235 steel by imidazoline derivative against $\mathrm{CO}_{2}$ corrosion," Corrosion Science, vol. 53, no. 1, pp. 353-361, 2011.

[44] G. Mu and X. Li, "Inhibition of cold rolled steel corrosion by Tween-20 in sulfuric acid: weight loss, electrochemical and AFM approaches," Journal of Colloid and Interface Science, vol. 289, no. 1, pp. 184-192, 2005.

[45] A. K. Satapathy, G. Gunasekaran, S. C. Sahoo, K. Amit, and P. V. Rodrigues, "Corrosion inhibition by Justicia gendarussa plant extract in hydrochloric acid solution," Corrosion Science, vol. 51, no. 12, pp. 2848-2856, 2009.

[46] E. E. Oguzie, Y. Li, and F. H. Wang, "Corrosion inhibition and adsorption behavior of methionine on mild steel in sulfuric acid and synergistic effect of iodide ion," Journal of Colloid and Interface Science, vol. 310, no. 1, pp. 90-98, 2007.

[47] L. M. Rodríguez-Valdez, W. Villamisar, M. Casales et al., "Computational simulations of the molecular structure and corrosion properties of amidoethyl, aminoethyl and hydroxyethyl imidazolines inhibitors," Corrosion Science, vol. 48, no. 12, pp. 4053-4064, 2006.

[48] H. Ju, Z. P. Kai, and Y. Li, “Aminic nitrogen-bearing polydentate Schiff base compounds as corrosion inhibitors for iron in acidic media: a quantum chemical calculation," Corrosion Science, vol. 50, no. 3, pp. 865-871, 2008.

[49] L. M. Rodríguez-Valdez, A. Martínez-Villafañe, and D. Glossman-Mitnik, "Computational simulation of the molecular structure and properties of heterocyclic organic compounds with possible corrosion inhibition properties," Journal of Molecular Structure: THEOCHEM, vol. 713, no. 1-3, pp. 65-70, 2005. 
[50] K. F. Khaled, "Molecular simulation, quantum chemical calculations and electrochemical studies for inhibition of mild steel by triazoles," Electrochimica Acta, vol. 53, no. 9, pp. 3484-3492, 2008.

[51] R. M. Issa, M. K. Awad, and F. M. Atlam, "Quantum chemical studies on the inhibition of corrosion of copper surface by substituted uracils," Applied Surface Science, vol. 255, no. 5, pp. 2433-2441, 2008. 



Submit your manuscripts at http://www.hindawi.com
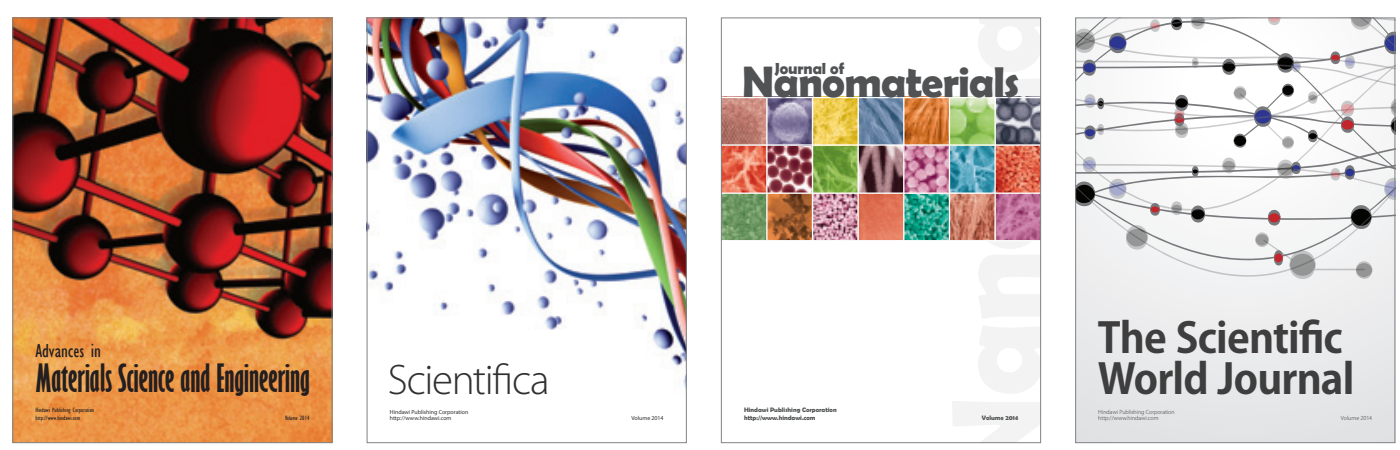

\section{The Scientific World Journal}
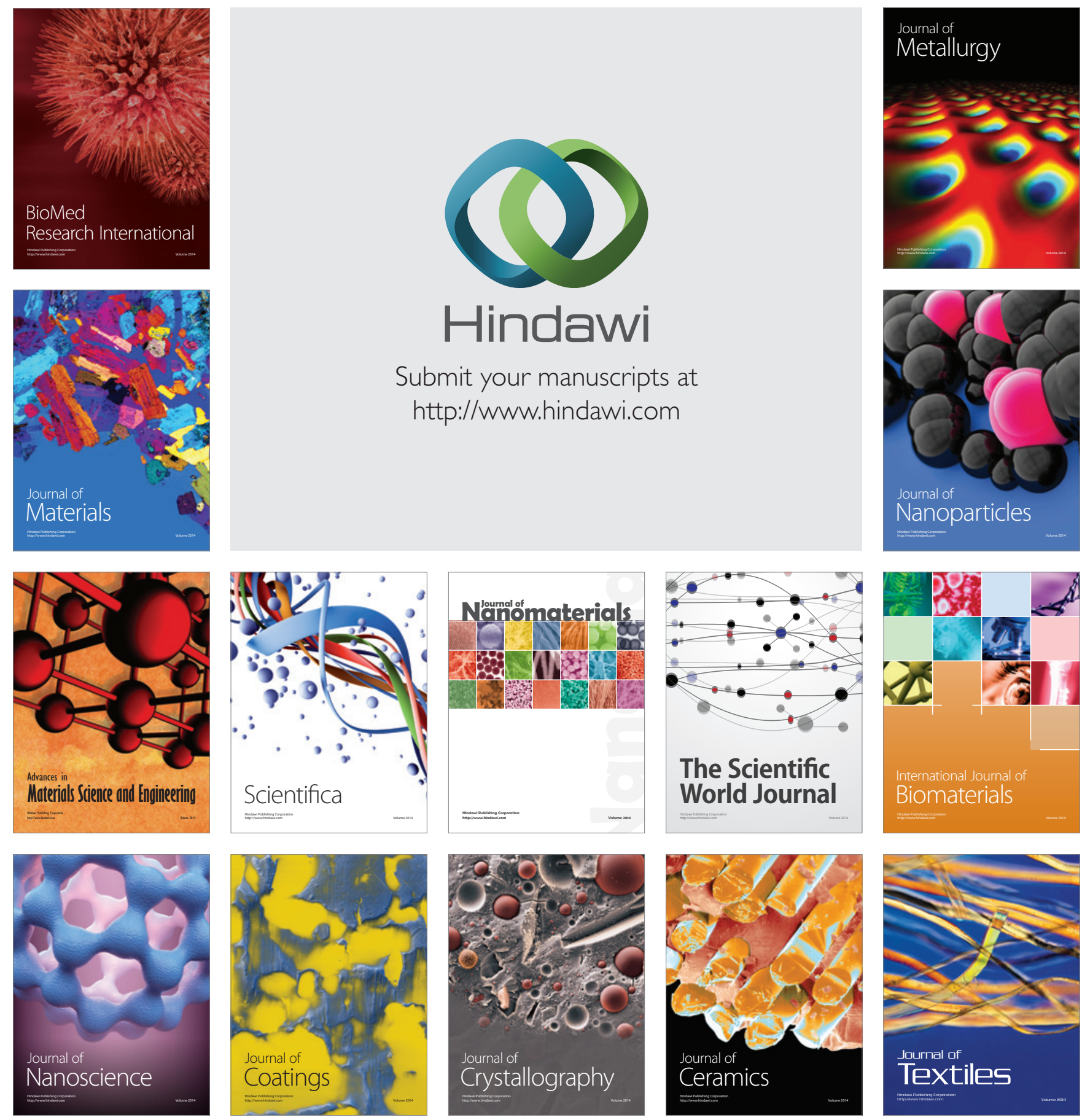